BMJ Paediatrics Open

\section{Retrospective review of paediatric case reports of Stevens-Johnson syndrome and toxic epidermal necrolysis with lamotrigine from an international pharmacovigilance database}

Oluwaseun Egunsola, ${ }^{1}$ Kristina Star, ${ }^{2,3}$ Kristina Juhlin, ${ }^{2}$ Sylvia H Kardaun, ${ }^{4}$ Imti Choonara, ${ }^{1}$ Helen M Sammons ${ }^{1,5}$
To cite: Egunsola 0, Star K, Juhlin $\mathrm{K}$, et al. Retrospective review of paediatric case reports of Stevens-Johnson syndrome and toxic epidermal necrolysis with lamotrigine from an international pharmacovigilance database. BMJ Paediatrics Open 2017;1:e000039. doi:10.1136/ bmjpo-2017-000039

- Prepublication history and additional material is available. To view please visit the journal (http://dx.doi.org/10.1136/ bmjpo-2017-000039).

Received 14 April 2017 Revised 15 June 2017 Accepted 16 June 2017

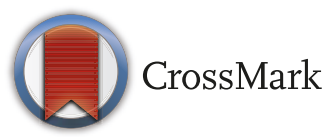

For numbered affiliations see end of article.

Correspondence to Dr Oluwaseun Egunsola; seunegunsola@yahoo.com

\section{ABSTRACT}

Objectives This study aims to characterise paediatric reports with lamotrigine (LTG) and Stevens-Johnson syndrome or toxic epidermal necrolysis (SJS/TEN), and to explore whether potential risk factors can be identified. Design This is a retrospective review of suspected adverse drug reaction (ADR) reports. Reported time from LTG start to SJS/TEN onset, indication for use and dose was explored. To identify potential risk groups, report features (eg, ages, patient sex, co-reported drugs) for LTG and SJS/TEN were contrasted with two reference groups in the same database, using shrinkage log0R.

Setting Reports were retrieved from VigiBase, the WHO global database of individual case safety reports, in January 2015.

Patients Data for patients aged $\leq 17$ years old were extracted.

Results There were 486 reports of SJS/TEN in LTGtreated paediatric patients. Ninety-seven per cent of the cases with complete information on time to onset of SJS/ TEN occurred within 8 weeks of initiation of LTG therapy. The median time to onset was 15 days (IQR: 10-22 days). The proportion of SJS/TEN with LTG and valproic acid (VPA) co-reporting was significantly more than non-cutaneous ADRs (43\% vs 19\%, (logOR: 1.60 (99\% Cl: 1.33 to 1.84)).

Conclusions The results suggest that VPA co-medication with LTG therapy is a risk factor for SJS/TEN in the paediatric population. Although this relationship has been identified from individual case reports, this is the first supportive study from a large compilation of cases. SJS/ TEN risk is highest in first 8 weeks of treatment with LTG in children and clinicians should be aware of this risk during this period.

\section{INTRODUCTION}

Stevens-Johnson syndrome (SJS) and toxic epidermal necrolysis (TEN) are acute mucocutaneous reactions characterised by generalised blistering, painful, target-like lesions on bright erythema. Patients typically present with prodromal symptoms, fever

\section{What is already known on this topic?}

Lamotrigine is a common cause of StevensJohnson syndrome and toxic epidermal necrolysis in paediatrics.

- The risk for Stevens-Johnson syndrome and toxic epidermal necrolysis from co-medication with lamotrigine and valproic acid has been described in individual case reports.

\section{What this study hopes to add?}

- This study presents a large dataset of globally collected reports on Stevens-Johnson syndrome/ toxic epidermal necrolysis and lamotrigine in paediatrics. The results strengthen previous findings.

- Stevens-Johnson syndrome/toxic epidermal necrolysis is most likely to occur in the first 8 weeks of lamotrigine treatment.

- Valproic acid and lamotrigine co-medication is a possible risk factor for Stevens-Johnson syndrome/ toxic epidermal necrolysis in children.

and severe mucositis. ${ }^{1}$ SJS and TEN belong to the same spectrum of disease with similar aetiological origin. TEN is the more severe of the two and is characterised by detached or detachable skin of $>30 \%$ of the total body surface area. SJS affects $<10 \%$ of the body surface area, while SJS/TEN overlap involves $10 \%-30 \%$ of the body surface area. ${ }^{2} \mathrm{~A}$ population-based study in Germany reported an incidence of 1.89 cases of SJS and TEN per million persons per year. ${ }^{3}$ Medicines are a common cause of SJS $/ \mathrm{TEN}^{4}$ with a highest risk of onset within the first 8 weeks of treatment. ${ }^{5}$ Mortality rates at 6 weeks have been reported to be between $7.5 \%$ and $23 \% .{ }^{67}$ 
The pathogenesis is not yet fully elucidated. However, both immunological and genetic factors are involved. Unmodified drugs or endogenously modified human leucocyte antigen (HLA)-bound drugs or their metabolites are presented to T cells to trigger immune response. ${ }^{8}$ Apoptotic keratinocyte death occurs via several pathways, for example, the ligation of Fas on keratinocyte by its ligand FasL on cytotoxic T cells or keratinocytes and the release of granulysin and other cytokines ${ }^{9}$ Blister fluids in TEN has been shown to contain large numbers of $\mathrm{T}$ lymphocytes, particularly CD8+ lymphocytes, which exhibit drug specific cytotoxicity. ${ }^{10}$

The rarity of SJS and TEN limits the practicability of large studies, particularly when investigating subpopulations and individual drugs. The highest number of paediatric cases so far published in the literature involved 80 patients. $^{71}$ Sulphonamides and antiepileptic drugs are the drugs most frequently suspected for causing SJS/ TEN. ${ }^{12}$ This study aims to characterise paediatric reports of cases of lamotrigine (LTG) associated with SJS/TEN from a large database of spontaneous reports and to explore whether potential risk factors can be identified and further analysed.

\section{METHOD}

\section{Data source and population}

A retrospective review of suspected adverse drug reaction (ADR) reports for the paediatric population with SJS or TEN following LTG treatment.

\section{VigiBase}

Individual case safety reports (ICSRs) of suspected ADRs from national pharmacovigilance centres worldwide are received by the Uppsala Monitoring Centre (UMC), WHO Collaborating Centre for International Drug Monitoring. ${ }^{13}$ In January 2015, about 120 countries participated in the WHO Programme for International Drug Monitoring and over 10 million individual reports had been submitted to VigiBase since its inception in 1968. These reports are stored in VigiBase, the WHO global database of ICSRs. The completeness and quality of information on the reports varies and the likelihood that the suspected adverse reaction is drug related is not the same for all cases. The ADRs are coded by the source countries either by using the WHO Adverse Reaction Terminology (WHO-ART) or the Medical Dictionary for Regulatory Activities (MedDRA). ${ }^{14}$

\section{Inclusion and exclusion of data}

A VigiBase search from inception to 1January 2015 was performed. An automatic duplicate detection algorithm was applied to exclude duplicate reports. ${ }^{1516}$ Only reports on paediatric ages were retrieved, defined as patients aged $\leq 17$ years old and further classified as neonates (0-27 days), infants (28 days to 23 months), children (2-11 years) and adolescents (12-17 years).
The primary dataset consisted of ICSRs with the WHODrug dictionary preferred base 'lamotrigine', noted by the reporter as the suspected or interacting drug, and reported with the MedDRA preferred terms (PT) ${ }^{14}$ 'Stevens-Johnson syndrome' or 'toxic epidermal necrolysis'. SJS/TEN overlap, however, is not a separate MedDRA listed PT and is reported either as SJS or as TEN.

The individual reports in the primary dataset were manually screened for duplicate reports and negative time to onset (TTO). Reports with a negative TTO, in which SJS/TEN occurred before LTG treatment was commenced, were excluded. Manually identified duplicates were verified by a second researcher before exclusion.

\section{Analysis}

\section{Overall data}

To identify potential risk groups, we contrasted report features for the paediatric population treated with LTG and experiencing SJS/TEN with two reference groups. The analysed features were as follows: age groups (defined earlier), patient sex, co-reported type of drugs (denoted suspected, interacting or concomitant), co-reported type of ADRs, reporting country/ geographical region and whether or not the cases were reported with fatal outcome.

The reference groups were as follows:

- Reports of LTG (suspected or interacting) recorded with any ADRs, except cutaneous ADRs.

- Reports with SJS/TEN with any suspected or interacting drug, except LTG.

The exclusion of cutaneous reports for reference group A was applied to avoid cases, overlapping with SJS/TEN, such as with erythema multiforme. Reports with cutaneous ADRs were defined with the MedDRA system organ class 'Skin and subcutaneous tissue disorders'. The disproportionality measure, OR, is used by UMC in its shrinkage version. ${ }^{17}$ Shrinkage $\log$ ORs were determined within vigiPoint. This is an analytic tool developed by the UMC that compares reported features for a defined set of reports with a reference set to identify key features in data. Shrinkage minimises the risk of identifying false associations in large-scale pattern discovery. ${ }^{17}$ The relative frequencies of reported features for LTG associated with SJS/TEN were contrasted to the reference datasets defined above, using ORs with adaptive statistical shrinkage. Significant key features were defined as those with $\log$ OR005 $>0.5$ or $\log$ OR $995<-0.5$, representing the lower limit or the upper limit of the $99 \%$ CI. Key features were defined as those features for the LTG and SJS/TEN dataset that were significantly different from both reference groups (A and B), with the exception of cutaneous reactions where comparison was only relevant to reference group B (since cutaneous reactions had been excluded in reference group A). 
Time to onset

Only reports with complete dates for start of LTG and onset of SJS/TEN were included in the TTO analysis. Reports with negative TTOs and those with estimated dates were excluded from this analysis. The number of days from start of LTG to onset of SJS/TEN was calculated. To investigate whether the TTO for reports with LTG and SJS/TEN corresponded to other antiepileptic agents known to cause these serious skin reactions, we also calculated the TTO for carbamazepine (CBZ) and phenobarbital (PBT). Log-rank test of the survival distribution SJS/TEN TTO with LTG indication and patients' age was carried out using the Kaplan-Meier survival analysis method.

\section{Dose}

For children< 13 years old, the dose of LTG $(\mathrm{mg} / \mathrm{kg} /$ day $)$ for each report was determined by dividing the daily dose $(\mathrm{mg})$ at the TTO of SJS/TEN by body weight $(\mathrm{kg})$. For those between 13 and 17 years old, daily LTG dose (mg/ day) was determined. Dosing data from all reports were summarised as median and IQR.

\section{RESULTS}

After automatic duplicate exclusion, we identified 500 paediatric cases of SJS/TEN where LTG was reported as suspected between 23 March 1994 (shortly after LTG approval in Europe) and 1 January 2015. Fourteen of these, including 12 duplicates identified after manual screening and 2 with unlikely attribution (negative TTO), were further excluded. The remaining 486 cases originating from 40 countries were eligible for inclusion. The identified key features in comparison with both reference groups are described in table 1.

LTG was co-reported with VPA in 207 (207/486, 43\%) of the SJS/TEN cases and administered alone in 158 of the SJS/TEN cases (158/486, 33\%), while 121 (121/486, $25 \%$ ) of the cases co-reported LTG with other drugs. There was a significantly higher proportion of patients with SJS/TEN receiving LTG with VPA than the proportion of patients with non-cutaneous ADRs receiving both drugs $(493 / 2609,19 \%)(\log O R: 1.60$ (1.33,1.84)). Similarly, a significantly higher proportion of patients with SJS/TEN received LTG with VPA than other drugs with VPA $(181 / 4349,4 \%)(\log$ OR: $3.63(3.37,3.88))$.

The median age for all patients was 12 years (IQR: $8-15$ years). Compared with non-cutaneous ADRs, cases of SJS/ TEN associated with LTG were rarely reported in infancy, similar in children and markedly higher in adolescence (figure 1). The proportion of SJS/TEN reports in adolescence was also significantly higher in patients receiving LTG than those without LTG. There were more female SJS/TEN cases $(277 / 482,57.5 \%)$ than male $(205 / 482$, $42.5 \%)$. This pattern deviated from the two reference groups, but was not significantly different according to the statistical thresholds used in this study (table 1). A total of 15 cases of fatality $(15 / 486,3 \%)$ and 25 cases $(25 / 486,5 \%)$ of recovery with sequelae were reported.

One hundred and ninety reports of SJS/TEN did not document the indication for LTG treatment. LTG was prescribed for the treatment of epilepsy in 69\% (203 cases) of the 296 cases with known indication; $60 \%$ of these cases were children and $38 \%$ were adolescents. Use for bipolar disorders and other forms of affective or behavioural disorders accounted for $31 \%$ (93 cases) of known indications. Fourteen per cent (13/93) of the cases receiving LTG for mood or behavioural disorders received concurrent VPA treatment compared with $54 \%$ (109/203) of the known cases of epilepsy receiving both drugs. Three quarter of the cases with affective disorders were adolescents and $25 \%$ were children.

Forty-eight per cent (232 cases) of the SJS/TEN reports with LTG provided sufficient information to determine the TTO of SJS/TEN (figure 2). In 97\%, SJS/TEN occurred within 8 weeks of initiation of LTG therapy. Three hundred and forty-two and 138 case reports of SJS / TEN with CBZ and PBT respectively provided information on the TTO and $95 \%$ and $96 \%$ of cases respectively occurred within 8 weeks. The median number of days between exposure to the drugs and the onset of SJS/ TEN was similar for all three drugs (LTG and SJS/TEN has a median TTO of 15 days (IQR: 10-22 days), CBZ and PBT were 14 days (IQR: 10-18 days) and 15 days (IQR: 10-22 days) respectively). The log-rank test of the survival distribution of SJS/TEN did not show any significant difference in the TTO between patients receiving LTG for epilepsy and mood disorders $(p=0.09)$. Similarly, there was no significant difference in TTO between children and adolescents $(\mathrm{p}=0.90)$.

Ninety-seven case reports $(20 \%)$ of SJS/TEN with LTG provided adequate information on LTG dosing, of which 38 patients received concomitant VPA medication. The median dose of LTG among children without VPA was $1.5 \mathrm{mg} / \mathrm{kg}$ (IQR: 1.1-2.8), while the median LTG dose with VPA was $1.4 \mathrm{mg} / \mathrm{kg}$ (IQR: 0.8-2.2). Among adolescents (13-17 years old) without concomitant VPA medication, the median LTG dose was $100 \mathrm{mg} /$ day (IQR: 50-150), while the median LTG dose with VPA was $50 \mathrm{mg} /$ day (IQR: 25-125).

\section{DISCUSSION}

This is the largest review of drug-associated SJS/TEN in paediatric patients based on a worldwide spontaneous reporting system, and therefore offers an opportunity to explore the effect of co-medication in this group. The study suggests that VPA co-medication increases the risk of SJS/TEN in paediatric patients receiving LTG. The proportion of reports of SJS/TEN associated with LTG and VPA was significantly higher than that of non-cutaneous ADRs. This is in keeping with a study in adults in which $74 \%$ and $64 \%$ of adults who developed SJS or TEN, respectively, received VPA co-medication with LTG. ${ }^{18}$ In concordance with an earlier multicentre European study, 


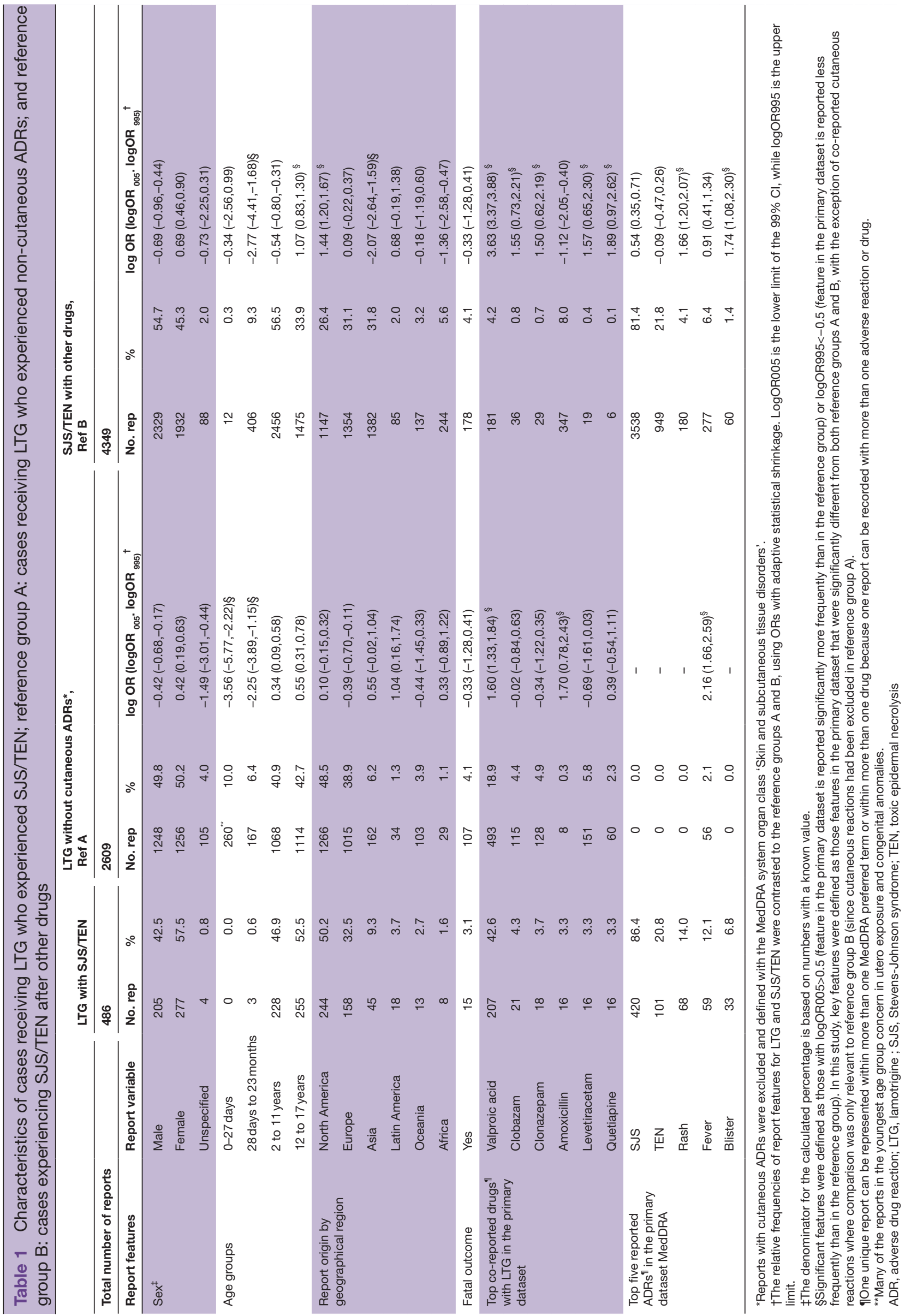




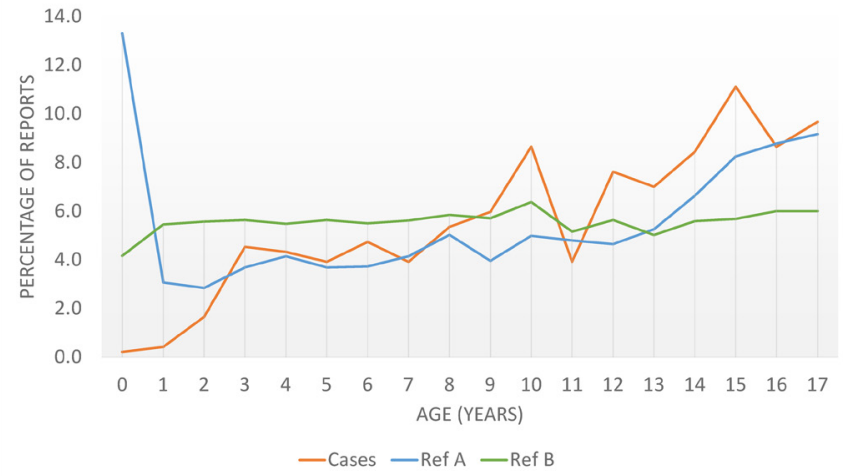

Figure 1 Percentage age distribution of lamotrigine-treated population who experienced Stevens-Johnson syndrome/ toxic epidermal necrolysis (cases), patients receiving lamotrigine who experienced non-cutaneous adverse drug reactions (reference A) and experienced Stevens-Johnson syndrome/toxic epidermal necrolysis after other drugs (reference B). Many of the reports for ages $<1$ year old concern in utero exposure and congenital anomalies.

SJS/TEN occurred mostly during the first 8 weeks of LTG exposure. ${ }^{5}$

LTG and VPA are very frequently co-prescribed for epilepsy because of their synergistic effect. ${ }^{19}$ The synergism between both drugs in patients with mood disorders has not been evaluated. As a result of its inhibitory effect on hepatic glucuronidation, VPA co-medication reduces LTG metabolism and plasma levels. In order to minimise the risk of ADRs due to high drug levels, lower initial and maintenance doses of LTG are recommended when prescribed with VPA. ${ }^{20}$ The effect of low dose or slow escalation of LTG on the risk of either SJS or TEN, when prescribed with VPA, could not be established in this study because dose data are limited. However, a lower median LTG dose was observed for children and adolescents receiving co-medication with VPA in a small subset of data in this study.

More than a quarter of the children and adolescents with SJS/TEN with recorded indication for use received LTG for affective and behavioural disorders. LTG is

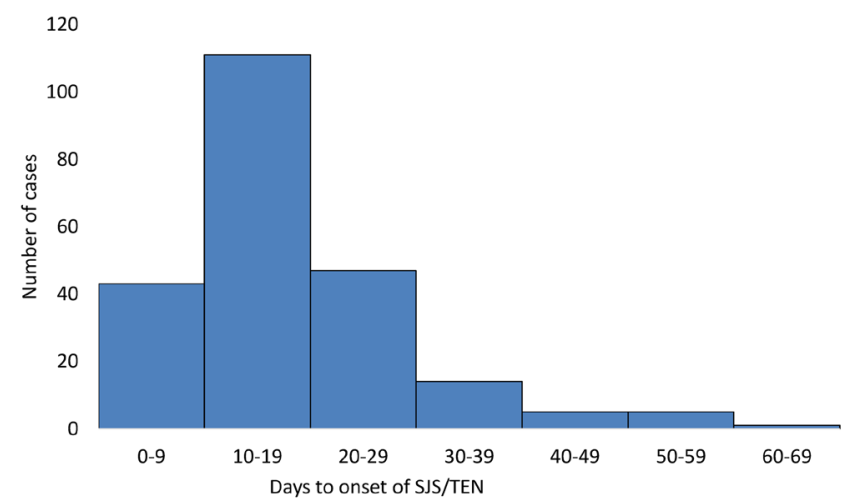

Figure 2 Time to onset of Stevens-Johnson syndrome (SJS) and toxic epidermal necrolysis (TEN) with lamotrigine. however not licensed for these conditions in children and adolescents, ${ }^{21}$ which means that these population of patients were treated without support from controlled randomised studies. Therefore, a careful risk-benefit assessment for the individual needs to be established before using LTG in this population.

\section{Limitations}

The likelihood that the suspected adverse reaction is drug related is not the same for all cases (see online Supplementary material). As previously mentioned, the effect of LTG dosing on SJS/TEN has not been determined and this could not be done in this study as a result of the marked heterogeneity in the reporting methods, as well as inadequate dosing information in several reports on VigiBase. Reporters were of a varied background, including doctors, pharmacists and consumers, and the reports varied in details. Furthermore, recording of TTO can vary, as some reporters might specify reaction onset as the date when the first symptoms started and others as the date of diagnosis. ${ }^{22}$ The list of co-medications in the reports may have also been incomplete. Although there is no baseline utilisation data to estimate the relative risk of SJS/TEN with the drugs, we have identified risks by using specific datasets in the database as comparators.

It is not impossible that cases of erythema multiforme and other forms of skin rash have been mispresented as SJS, particularly in the older reports. ${ }^{23}$ However, we consider the influence from these limitations on the core analysis to be marginal, since the study focused on comparing report features for LTG and SJS/TEN with two reference groups which were subject to the same limitations. The number of fatal cases or long-term sequelae from SJS/TEN following LTG treatment was low possibly because late sequelae may have been missed, as follow-up was often not reported for the cases.

\section{Conclusions}

LTG co-medication with VPA is a possible risk factor for $\mathrm{SJS} / \mathrm{TEN}$, and these risks are generally restricted to the first 8 weeks of treatment with LTG. A cohort study is required to further establish this risk. Clinicians should be aware of these, and parents and children should be counselled accordingly.

\section{Author affiliations \\ ${ }^{1}$ Division of Medical Sciences and Graduate Entry Medicine, School of Medicine, University of Nottingham, Derby, UK \\ ${ }^{2}$ Uppsala Monitoring Centre, WHO Collaborating Centre for International Drug Monitoring, Uppsala, Sweden \\ ${ }^{3}$ Department of Public Health and Caring Sciences, Uppsala University, Uppsala, Sweden \\ ${ }^{4}$ Department of Dermatology, Reference Center for Cutaneous Adverse Reactions, University Medical Center Groningen, University of Groningen, Groningen, The Netherlands \\ ${ }^{5}$ North Devon District Hospital, Raleigh Park, Barnstaple, UK}

Acknowledgements The authors are indebted to the National Pharmacovigilance Centres that contribute data to VigiBase. The opinions and conclusions in this study are not necessarily those of the various centres or of the WHO. 
Contributors $\mathrm{OE}, \mathrm{IC}, \mathrm{HMS}, \mathrm{KS}$ and $\mathrm{KJ}$ conceptualized and designed the study, and drafted the initial manuscript. SHK reviewed and revised the subsequent versions of the manuscript and all authors approved the final manuscript as submitted.

\section{Competing interests None declared.}

Provenance and peer review Commissioned; externally peer reviewed.

Open Access This is an Open Access article distributed in accordance with the Creative Commons Attribution Non Commercial (CC BY-NC 4.0) license, which permits others to distribute, remix, adapt, build upon this work non-commercially, and license their derivative works on different terms, provided the original work is properly cited and the use is non-commercial. See: http://creativecommons.org/ licenses/by-nc/4.0/

(c) Article author(s) (or their employer(s) unless otherwise stated in the text of the article) 2017. All rights reserved. No commercial use is permitted unless otherwise expressly granted.

\section{REFERENCES}

1. Harr T, French LE. Toxic epidermal necrolysis and Stevens-Johnson syndrome. Orphanet J Rare Dis 2010;5:39.

2. Bastuji-Garin S, Rzany B, Stern RS, et al. Clinical classification of cases of toxic epidermal Necrolysis, Stevens-Johnson syndrome, and erythema multiforme. Arch Dermatol 1993;129:92-6.

3. Rzany B, Mockenhaupt M, Baur S, et al. Epidemiology of erythema exsudativum multiforme Majus, Stevens-Johnson syndrome, and toxic epidermal necrolysis in Germany (1990-1992): structure and results of a population-based registry. $J$ Clin Epidemiol 1996:49:769-73.

4. Mockenhaupt M, Viboud C, Dunant A, et al. Stevens-Johnson syndrome and toxic epidermal necrolysis: assessment of medication risks with emphasis on recently marketed drugs. the EuroSCARstudy. J Invest Dermatol 2008;128:35-44.

5. Rzany B, Correia O, Kelly JP, et al. Risk of Stevens-Johnson syndrome and toxic epider mal necrolysis during first weeks of antiepileptic therapy: a case-control study. The Lancet 1999:353:2190-4.

6. Sekula P, Dunant A, Mockenhaupt M, et al. Comprehensive survival analysis of a cohort of patients with Stevens-Johnson syndrome and toxic epidermal necrolysis. J Invest Dermatol 2013;133:1197-204.

7. Levi N, Bastuji-Garin S, Mockenhaupt M, et al. Medications as risk factors of Stevens-Johnson syndrome and toxic epidermal necrolysis in children: a pooled analysis. Pediatrics 2009;123:e29 7-e304.

8. Chung WH, Wang CW, Dao RL. Severe cutaneous adverse drug reactions. J Dermatol 2016;43:758-66.

9. Abe R. Cutaneous adverse drug reactions: Stevens-Johnson Syndrome and Toxic Epidermal Necrolysis. Immunology of the Skin 2016:393-404.

10. Mockenhaupt M. The current understanding of Stevens-Johnson syndrome and toxic epidermal necrolysis. expert review of clinical immunology. Expert Rev Clin Immunol 2011;7:803-13.

11. Ferrandiz-Pulido C, Garcia-Patos V. A review of causes of StevensJohnson syndrome and toxic epidermal necrolysis in children. Arch Dis Child 2013;98:998-1003.

12. Roujeau JC, Kelly JP, Naldi L, et al. Medication use and the risk of Stevens-Johnson syndrome or toxic epidermal necrolysis. N Engl $J$ Med 1995;333:1600-8.

13. VigiBase LM. The WHO global ICSR database system: basic facts. Drug Inf J 2008;42:409-19.

14. Medical Dictionary for Regulatory activities. http://www.meddra.org/.

15. Nor?n GN, Orre R, Bate A, et al. Duplicate detection in adverse drug reaction surveillance. Data Min Knowl Discov 2007;14:305-28.

16. Tregunno PM, Fink DB, Fernandez-Fernandez C, et al. Performance of probabilistic method to detect duplicate individual case safety reports. Drug Saf 2014;37:249-58.

17. Norén GN, Hopstadius J, Bate A. Shrinkage observed-to-expected ratios for robust and transparent large-scale pattern discovery. Stat Methods Med Res 2013;22:57-69.

18. Schlienger RG, Shapiro LE, Shear NH. Lamotrigine-induced severe cutaneous adverse reactions. Epilepsia 1998;39 Suppl 7:S22-S26.

19. Brodie MJ, Yuen AW, Group S. Lamotrigine substitution study: evidence for synergism with sodium valproate? 105 Study Group. Epilepsy Res 1997;26:423-32.

20. Anderson GD, Yau MK, Gidal BE, et al. Bidirectional interaction of valproate and lamotrigine in healthy subjects. Clin Pharmacol Ther 1996;60:145-56

21. Hart SR, Brock SE, Identifying JI. Assessing, and treating bipolar disorder at School. Springer US 2014.

22. Klepper MJ, Edwards B. Individual case safety reports--how to determine the onset date of an adverse reaction: a survey. Drug Saf 2011;34:299-305.

23. Tay YK, Huff JC, Weston WL. Mycoplasma pneumoniae infection is associated with Stevens-Johnson syndrome, not erythema multiforme (von Hebra). J Am Acad Dermatol 1996;35:757-60. 